\title{
Power Supply Synchronization without Communication
}

\author{
Leonardo A. B. Tôrres*, João P. Hespanha ${ }^{\dagger}$, Jeff Moehlis ${ }^{\dagger}$
}

\begin{abstract}
We consider the synchronization of power supplies in an isolated grid with multiple small-to-medium power sources. We show how to achieve a coordinated or synchronized behavior by imposing suitable dynamical behaviors to the controlled converters that interface each power source with the local power bus, without any explicit exchange of information among the power supply units. This approach avoids the need for an independent communication network and therefore improves the overall system reliability. The method proposed to achieve synchronization does not rely on the common approach of mimicking the dynamical behavior of interconnected synchronous generators.
\end{abstract}

\section{INTRODUCTION}

The problem of interconnecting multiple power supplies in parallel to provide energy to a commom load arises, e.g., in the analysis of power plant generators interconnected by a common electrical network in Wide Area Electrical Power Systems - WAEPS [1]; in local area power systems interconnections based on distributed generation, known as Micro-Grid based Electrical Power Systems - MGEPS [2]; and in Parallel Connected Uninterruptible Power Supplies - PCUPS [3].

A key consideration in building an isolated power system fed by multiple power supplies is the need to allow for power units to be added as the power grows and to replace and/or repair existing units without compromising the whole system. To accomplish this in a reliable fashion, the power units should use control strategies for voltage synchronization that rely solely on variables that can be measured locally, thus avoiding the need for a dedicated communication channel between units. Such a communication channel could become a single point-of-failure for the power system and severely compromise the overall system reliability.

Classical methods for the analysis of WAEPS show that, for appropriate system parameters, a newly added power plant generator naturally synchronizes with a previously synchronized ensemble of power generators, as long as its initial phase is close to that of the ensemble. The reader is referred to [4] for references to classical work in this area, as well as a new treatment of this problem that relates power synchronization with non-uniform Kuramoto oscillators, obviating some limitations of previous analysis methods.

In this paper, we are especially interested in MGEPS and PCUPS that differ from WEAPS in that there is significant

\footnotetext{
*Corresponding author: torres@cpdee.ufmg.br. L. Torres is with the Department of Electronic Engineering, Universidade Federal de Minas Gerais, Brazil.

$\dagger$ J. Hespanha and J. Moehlis are with the Departments of Electrical and Computer Engineering and Mechanical Engineering, respectively, University of California, Santa Barbara, USA
}

room to design the dynamics of each power unit because in MGEPS and PCUPS the voltage power supplies are implemented as highly efficient power electronic devices (typically, inverters) that interface the primary energy sources with the power bus, as depicted in Figure 1. Since these power electronic devices are capable of high switching frequencies, exhibit very fast responses, and can be endowed with significant computational power, sophisticated strategies can be adopted for voltage synchronization.

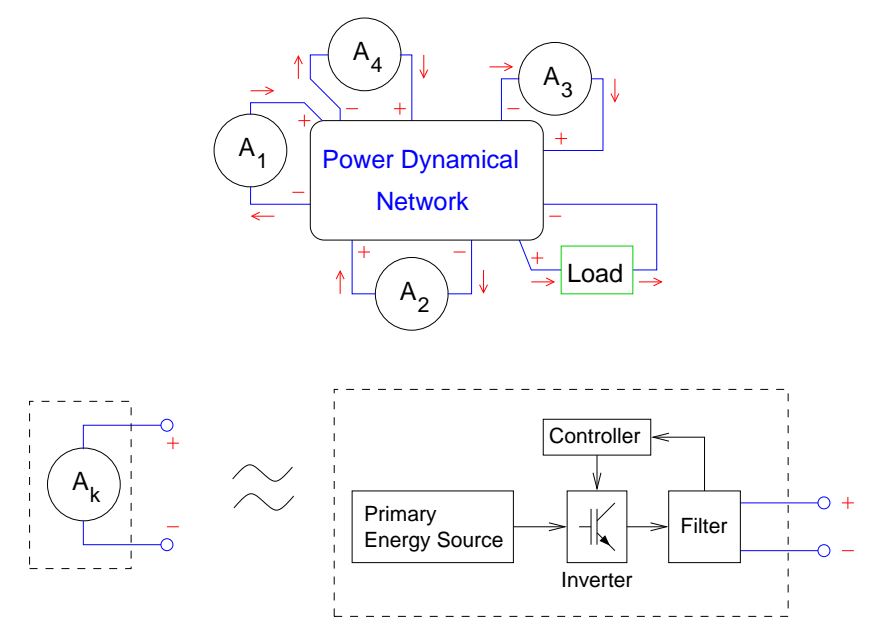

Figure 1. Voltage power sources represented as controlled nonlinear oscillators $A_{k}, k=1,2,3,4$, that should attain synchronization as a byproduct of delivering power to the same load through a reactive electrical network, without any explicit communication among the units.

Despite the potential flexibility of power electronic inverters, their parallel operation without a dedicated communication channel is still heavily influenced by the solutions adopted in the WAEPS domain. One such popular strategy is the socalled Voltage and Frequency Droop Method [5], in which each inverter unit varies its frequency according to the active power provided to the load, while the voltage amplitude is varied depending on the reactive power delivered to the load. This method can be traced back to [6] and to the more recent papers [7-9]. As the method relies on average delivered power (active and reactive) and exploits the natural time-scale separation of the problem, the correponding overall synchronization dynamics tends to be very slow when compared to the period of the voltage signals in steady state.

The main objective of the present paper is to show that the coordination of interconnected voltage power sources without explicit interchange of information can be regarded as a problem of dynamical systems synchronization, opening the path for augmented stability and faster synchronization 
response. Our preliminary results demonstrate the promise of this approach.

The results presented here are inspired by results in [10 12], where the concepts of passivity and incremental passivity are used to develop synchronization strategies. The use of passivity to study the synchronization of voltage power supplies through the excitation of a common electrical network is especially attractive because:

1) Linear electrical networks made of passive components are naturally passive systems - also known as positive real systems [13] - and there is a vast literature on the analysis of such systems [14].

2) Passivity properties are relatively robust with respect to variations in system parameters variations, leading to structurally robust analysis and design methods.

3) Passivity is naturally associated with input-output system representations, which facilitates the analysis of large systems through its decomposition into smaller subsystems.

An important novel feature of our work is that we go beyond the results in [10-12] by considering interconnection structures that have two peculiar characteristics usually not found in the literature on synchronization:

1) The interconnection structure is such that the different subsystems do not become decoupled as they become synchronized.

2) The interconnection structure is a dynamical system per se, and cannot be described by static algebraic relations associated with an underlying graph structure.

The remainder of this paper is organized as follows: Section II summarizes the key elements of the system under consideration, namely the refence models for primary energy sources with associated inverters, and the electrical network that connects them. Section III describes our approach to analyse the interconnected systems and states the main theoretical result, which states that the different power sources will synchronize asymptotically. Numerical simulations illustrating the effectiveness of the proposed designs are provided in Section IV, and some final conclusions and directions for future research are provided in Section V.

\section{NONLINEAR OSCILlators AND PASSIVE ElECTRICAL NETWORKS}

To investigate the ideas presented in Section I, we assume that each primary energy source and the associated inverter behaves as a nonlinear oscillator represented by a given reference model. These oscillators react solely to the local variables (voltage and current) measured at the power unit (Figure 1). All reference models are assumed equal, but depart from distinct (non-synchronized) initial conditions.

The electrical network (including the load) is a Linear Time-Invariant system with inductors, resistors, capacitors, and multi-linear transformers, and is therefore a passive system [14] that can be represented by a transfer function matrix $G(s)$. We assume that the electrical network is symmetric from the point of view of its access ports, i.e., all power supplies see the same transfer function from their ports to any other ports.
This means that the transfer matrix $G(s)$ has the following structure:

$$
\begin{aligned}
G(s) & =\left[\begin{array}{cccc}
g_{\mathrm{d}}(s) & g_{\mathrm{nd}}(s) & \ldots & g_{\mathrm{nd}}(s) \\
g_{\mathrm{nd}}(s) & g_{\mathrm{d}}(s) & \ldots & g_{\mathrm{nd}}(s) \\
\vdots & \vdots & \ddots & \vdots \\
g_{\mathrm{nd}}(s) & g_{\mathrm{nd}}(s) & \ldots & g_{\mathrm{d}}(s)
\end{array}\right] \\
& =\left(g_{\mathrm{d}}(s)-g_{\mathrm{nd}}(s)\right) I_{N}+g_{\mathrm{nd}}(s) \overrightarrow{1}^{\top}
\end{aligned}
$$

where $g_{\mathrm{d}}(s)$ and $g_{\text {nd }}(s)$ are rational transfer functions. This structure corresponds to a start-shape like the one in Figure 2 with equal connecting admittances $g_{1}(s)=g_{2}(s)=\ldots=$ $g_{N}(s)=g_{\mathrm{b}}(s)$ and a transfer matrix given by

$$
G(s)=g_{\mathrm{b}}(s) I_{N}-\frac{\left[g_{\mathrm{b}}(s)\right]^{2}}{N g_{\mathrm{b}}(s)+g_{\mathrm{L}}(s)} \overrightarrow{1}^{\top},
$$

where $g_{\mathrm{L}}(s)$ is the rational transfer function corresponding to the load admittance.

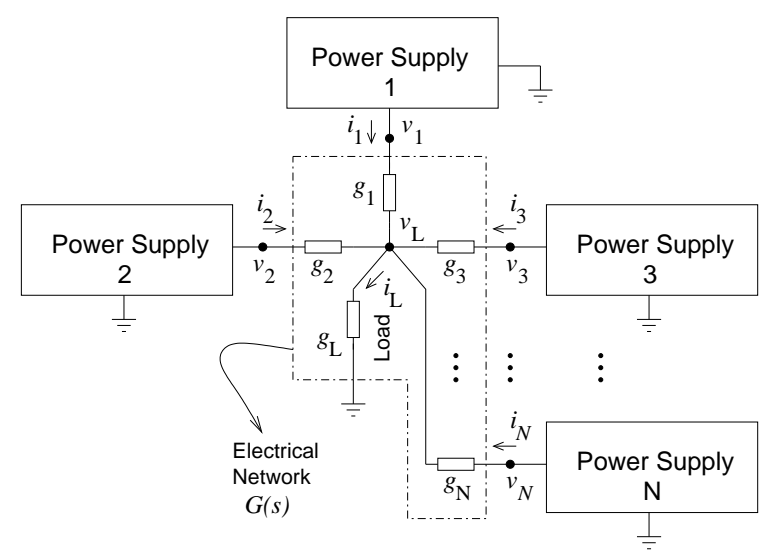

Figure 2. An overview of the specific problem investigated in this paper. The network that interconnects the power supplies is considered to be symmetric and typically contains one or several loads (admitance $g_{\mathrm{L}}$ in the figure) and several connecting components (admitances $g_{1}=g_{2}=\ldots g_{N}$ in the figure).

In the following development a more stringent condition will be assumed, namely that the electrical network is Input Strictly Passive (ISP) [13], i.e., that there exists a differentiable positive definite scalar function $S_{\mathrm{n}}(\cdot): \mathbb{R}^{m} \rightarrow \mathbb{R}^{+}$, such that

$$
\dot{S}_{\mathrm{n}}\left(\vec{x}_{\mathrm{n}}(t)\right) \leq-\eta \vec{u}_{\mathrm{n}}^{\top} \vec{u}_{\mathrm{n}}+\vec{y}_{\mathrm{n}}^{\top} \vec{u}_{\mathrm{n}}
$$

for some $\eta>0$. In the frequency domain, the ISP property is characterized by the following conditions:

1) All poles of $G(s)$ have strictly negative real parts;

2) There exists a scalar $\delta$ such that $G(j \omega)+G^{\top}(-j \omega) \geq$ $\delta>0$ for all $\omega \in \mathbb{R} \cup\{-\infty,+\infty\}$.

The first condition implies that the electrical network is an asymptotically stable LTI system, while the second requirement implies that the realization

$$
\begin{aligned}
& \dot{\vec{x}}_{\mathrm{n}}=A_{\mathrm{n}} \vec{x}_{\mathrm{n}}+B_{\mathrm{n}} \vec{u}_{\mathrm{n}}, \\
& \vec{y}_{\mathrm{n}}=C_{\mathrm{n}} \vec{x}_{\mathrm{n}}+D_{\mathrm{n}} \vec{u}_{\mathrm{n}}
\end{aligned}
$$

is such that $D_{\mathrm{n}}+D_{\mathrm{n}}^{\top} \geq \delta>0$, where $\vec{u}_{\mathrm{n}}=$ $\left[\begin{array}{llll}u_{1}^{\mathrm{n}} & u_{2}^{\mathrm{n}} & \ldots & u_{N}^{\mathrm{n}}\end{array}\right]^{\top} \in \mathbb{R}^{N}$ is the vector of voltages at the input ports of the electrical network, i.e. $u_{k}^{\mathrm{n}}=v_{k}$ in Figure 2, with $k=1,2, \ldots, N ; \vec{y}_{\mathrm{n}}=\left[\begin{array}{llll}y_{1}^{\mathrm{n}} & y_{2}^{\mathrm{n}} & \ldots & y_{N}^{\mathrm{n}}\end{array}\right]^{\top} \in \mathbb{R}^{N}$ is the vector 
of currents flowing into the network as a result of the applied voltages, such that $y_{k}^{\mathrm{n}}=i_{k}$ in Figure 2 .

\section{A. Nonlinear Oscillator Reference Model}

Inspired by the results in [11], we propose the following three step construction to design the reference model for each power supply:

1) Start with a Linear Time-Invariant (LTI) Single-Input Single-Output (SISO) dynamical system described by

$$
\begin{aligned}
\dot{\vec{x}}_{k} & =A_{\mathrm{a}} \vec{x}_{k}+B_{\mathrm{a}} \tilde{u}_{k}, \quad k \in\{1,2, \ldots, N\}, \\
y_{k} & =C_{\mathrm{a}} \vec{x}_{k},
\end{aligned}
$$

where $\vec{x}_{k} \in \mathbb{R}^{n} ; \tilde{u}_{k} \in \mathbb{R} ; y_{k} \in \mathbb{R}$; with the pair $\left(C_{\mathrm{a}}, A_{\mathrm{a}}\right)$ detectable. In addition, each LTI system should satisfy the following dissipation inequality:

$$
\dot{S}_{k}^{\mathrm{a}}\left(\vec{x}_{k}\right) \leq-\alpha_{\text {passive }}^{*} y_{k}^{2}+\tilde{u}_{k} y_{k}
$$

where $S_{k}^{\mathrm{a}}(\cdot): \mathbb{R}^{n} \rightarrow \mathbb{R}^{+}$is a continuously differentiable positive definite storage function, and $\alpha_{\text {passive }}^{*}>0$. This dissipation inequality qualifies the LTI as an Output Strictly Passive (OSP) system [13]. Notice that the combination of OSP and detectability guarantees that each LTI SISO system is globally asymptotically stable.

2) Introduce the following "internal" destabilizing feedback connection to (4):

$$
\tilde{u}_{k}=u_{k}^{\mathrm{a}}-\phi_{\alpha}\left(y_{k}, L_{k}\right)
$$

where $u_{k}^{\mathrm{a}}, k=1,2, \ldots, N$, corresponds to the current flowing into the power supply unit $k$ (in Figure 1, $\left.u_{k}^{\mathrm{a}}=-i_{k}\right)$; and $\phi_{\alpha}(\cdot): \mathbb{R} \rightarrow \mathbb{R}$ is a saturation type nonlinearity (see Figure 3 ) described by

$$
\phi_{\alpha}\left(y_{k}, L_{k}\right)=\left\{\begin{aligned}
-\alpha y_{k}, & \text { if }\left|y_{k}\right|<L_{k}, \\
-\alpha L_{k}, & \text { if } y_{k} \geq L_{k}, \\
\alpha L_{k}, & \text { if } y_{k} \leq-L_{k},
\end{aligned}\right.
$$

with $\alpha>\alpha_{\text {passive }}^{*}>0$, and $L_{k} \equiv L_{k}(t) \geq 0, k=$ $1,2, \ldots, N, \forall t \geq 0$, is a time-varying breakpoint.

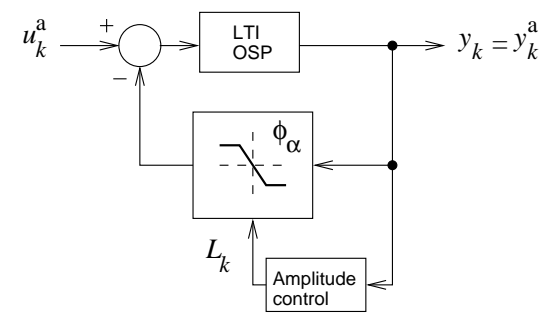

Figure 3. Graphical representation of the nonlinear oscillator used as a reference model for each power supply unit.

3) Add the following proportional-integral - PI control law as an adaptation strategy to determine the breakpoints $L_{k}(t)$, such that its value can be used to keep the RMS output voltage of each power supply unit equal to a given reference value $v_{\text {ref }}\left(\right.$ e.g. $\left.v_{\text {ref }}=120 \mathrm{~V}\right)$ :

$$
\begin{aligned}
\dot{\xi}_{k} & =\frac{1}{\tau}\left[-\xi_{k}+\left(y_{k}\right)^{2}\right], \\
\dot{\zeta}_{k} & =e_{k}^{\mathrm{v}}, \\
e_{k}^{\mathrm{v}} & =v_{\mathrm{ref}}-\sqrt{\left|\xi_{k}\right|} ; \\
L_{k}(t) & =\max \left\{0 ; K_{\mathrm{p}} e_{k}^{\mathrm{v}}+K_{\mathrm{i}} \zeta_{k}\right\}
\end{aligned}
$$

where $k=1,2, \ldots, N ; \sqrt{\left|\xi_{k}\right|} \geq 0$ is the estimated rootmean-square - RMS output voltage of the power supply $k$, which is obtained using $\tau$ sufficiently greater than the nominal period $T_{\text {nom }}$ expected for the voltage signals from the power supplies in steady-state (e.g. $T_{\text {nom }}=$ $1 / 60 \mathrm{~Hz}=16.66 \mathrm{~ms}) . \zeta_{k} \in \mathbb{R}$ is the integral of the difference between the RMS reference voltage $v_{\text {ref }}$ and the estimated output RMS voltage of the power supply $k$; and $K_{\mathrm{p}}, K_{\mathrm{i}}$ are the parameters of the PI controllers used in all power supply units.

Following the above construction, each power supply unit can become an amplitude controlled nonlinear oscillator. To accomplish this, the parameter $\alpha>\alpha_{\text {passive }}^{*}>0$ in (7) must be chosen sufficiently large to overcome the natural energy dissipation in the electrical network and to guarantee the existence of sustained oscillations.

Notice that, despite the fact that an oscillator cannot be a passive system since it is unstable in the Lyapunov sense, it can still be incrementally passive, as discussed below.

\section{SyNChRONIZATION AND INCREMENTAL PASSIVITY}

A dynamical system represented by

$$
\begin{aligned}
& \dot{\vec{x}}=f(\vec{x}, \vec{u}), \\
& \vec{y}=h(\vec{x}, \vec{u}),
\end{aligned}
$$

is said to be Incrementally Input Strictly Passive (IISP) (resp. Incrementally Output Strictly Passive (IOSP)) if and only if there exists a positive definite incremental storage function $S_{\Delta}^{\mathrm{ISP}}(\Delta \vec{x})$ and $\mu>0$ (resp. $S_{\Delta}^{\mathrm{OSP}}(\Delta \vec{x})$ and $\gamma>0$ ) such that the following respective inequalities hold, $\forall t \geq 0$ :

$$
\begin{aligned}
\dot{S}_{\Delta}^{\mathrm{ISP}}(\Delta \vec{x}) \leq- & \mu\left\|\vec{u}^{1}(t)-\vec{u}^{2}(t)\right\|^{2} \\
& +\left[\vec{u}^{1}(t)-\vec{u}^{2}(t)\right]^{\top}\left[\vec{y}^{1}(t)-\vec{y}^{2}(t)\right], \\
\dot{S}_{\Delta}^{\mathrm{OSP}}(\Delta \vec{x}) \leq- & \gamma\left\|\vec{y}^{1}(t)-\vec{y}^{2}(t)\right\|^{2} \\
& +\left[\vec{u}^{1}(t)-\vec{u}^{2}(t)\right]^{\top}\left[\vec{y}^{1}(t)-\vec{y}^{2}(t)\right],
\end{aligned}
$$

with $\Delta \vec{x}=\vec{x}\left(t ; \vec{x}_{0}^{1}, \vec{u}^{1}\right)-\vec{x}\left(t ; \vec{x}_{0}^{2}, \vec{u}^{2}\right)$ such that the timederivatives in the left-hand side are taken along the difference between the solutions $\vec{x}\left(t ; \vec{x}_{0}^{1}, \vec{u}^{1}\right)$ and $\vec{x}\left(t ; \vec{x}_{0}^{2}, \vec{u}^{2}\right)$ to (9) starting, respectively, at $\vec{x}(0)=\vec{x}_{0}^{1}$ with input $\vec{u}^{1}(t)$ and output $\vec{y}^{1}(t)$, and at $\vec{x}(0)=\vec{x}_{0}^{2}$ with input $\vec{u}^{2}(t)$ and output $\vec{y}^{2}(t)$.

The importance of these inequalities relies on the fact that they can be used to investigate if there is convergent behavior among all possible trajectories of the system corresponding to different initial conditions and inputs, which is instrumental in the context of synchronization of multiple dynamical systems described by the same set of differential equations. 
Despite the fact that the above incremental inequalities represent strong relations, for LTI systems of the form

$$
\begin{aligned}
& \dot{\vec{x}}=A \vec{x}+B \vec{u}, \\
& \vec{y}=C \vec{x}+D \vec{u},
\end{aligned}
$$

the properties of Incremental ISP and Incremental OSP are equivalent to the ISP and OSP properties. This can be seen by noticing that the above equations remain true after the following substitution of variables: $\vec{x} \sim \Delta \vec{x}, \vec{u} \sim \Delta \vec{u}=$ $\vec{u}^{1}(t)-\vec{u}^{2}(t)$, and $\vec{y} \sim \Delta \vec{y}=\vec{y}^{1}(t)-\vec{y}^{2}(t)$. This shows that any dissipation inequality that is true for the LTI system will have its incremental counterpart after applying this same change of variables. It is interesting to notice that, in this case, an incremental storage function can be easily obtained through the substitution $\vec{x} \sim \Delta \vec{x}$ in the corresponding storage function.

Specifically, from the ISP dissipation inequality (2) associated to the interconnection structure, and considering the previous discussion, we have that

$$
\dot{S}_{\mathrm{n}}\left(\Delta \vec{x}_{\mathrm{n}}\right) \leq-\eta\left\|\Delta \vec{u}_{\mathrm{n}}\right\|^{2}+\Delta \vec{y}_{\mathrm{n}}^{\top} \Delta \vec{u}_{\mathrm{n}},
$$

with $\Delta \vec{u}_{\mathrm{n}}=\vec{u}_{\mathrm{n}}^{1}(t)-\vec{u}_{\mathrm{n}}^{2}(t)$, and $\Delta \vec{y}_{\mathrm{n}}=\vec{y}_{\mathrm{n}}^{1}(t)-\vec{y}_{\mathrm{n}}^{2}(t)$. However, to guarantee synchronization among the power supply units based on energy dissipation effected by the electrical network, when the units are not synchronized, it is interesting to search for the maximum incremental dissipation associated with the differences between output voltages. In this context, we want to look for an incremental dissipation inequality similar to the above, such that

$$
\dot{S}_{\mathrm{n}}\left(\Delta \vec{x}_{\mathrm{n}}\right) \leq-\eta_{\mathrm{s}}\left\|\Delta \vec{u}_{\mathrm{n}}\right\|^{2}+\Delta \vec{y}_{\mathrm{n}}^{\top} \Delta \vec{u}_{\mathrm{n}}, \quad \eta_{\mathrm{s}} \geq \eta .
$$

On the other hand, from (5) and (6), the OSP property of the linear part of the nonlinear reference model (Section II-A) implies that

$$
\dot{S}_{\mathrm{a}}\left(\vec{x}_{\mathrm{a}}\right) \leq\left(\alpha-\alpha_{\text {passive }}^{*}\right) \vec{y}_{\mathrm{a}}^{\top} \vec{y}_{\mathrm{a}}+\vec{y}_{\mathrm{a}}^{\top}\left[\vec{u}_{\mathrm{a}}-\vec{u}_{\alpha}\right],
$$

where $S_{\mathrm{a}}\left(\vec{x}_{\mathrm{a}}\right)=S_{1}^{\mathrm{a}}\left(\vec{x}_{1}\right)+S_{2}^{\mathrm{a}}\left(\vec{x}_{2}\right)+\cdots+S_{N}^{\mathrm{a}}\left(\vec{x}_{N}\right) ; \vec{u}_{\mathrm{a}}=$ $\left[u_{1}^{\mathrm{a}} u_{2}^{\mathrm{a}} \ldots u_{N}^{\mathrm{a}}\right]^{\top} \in \mathbb{R}^{N}$, and

$$
\vec{u}_{\alpha}=\left[\psi_{\alpha}\left(y_{1}, L_{1}\right) \psi_{\alpha}\left(y_{2}, L_{2}\right) \ldots \psi_{\alpha}\left(y_{N}, L_{N}\right)\right]^{\top} \in \mathbb{R}^{N},
$$

is a vector of nondecreasing, dead-zone type, nonlinear functions (see Figure 4):

$$
\psi_{\alpha}\left(y_{k}, L_{k}\right)=\phi_{\alpha}\left(y_{k}, L_{k}\right)+\alpha y_{k} .
$$

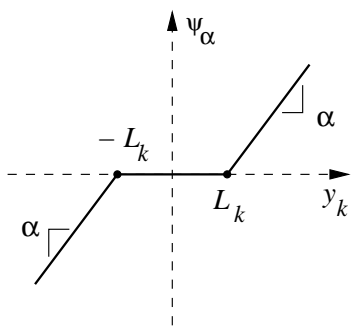

Figure 4. Dead-zone type nonlinearity corresponding to (12).

Similarly to (10), the incremental version of inequality (11) corresponds to

$$
\dot{S}_{\mathrm{a}}\left(\Delta \vec{x}_{\mathrm{a}}\right) \leq\left(\alpha-\alpha_{\text {passive }}^{*}\right)\left\|\Delta \vec{y}_{\mathrm{a}}\right\|^{2}+\Delta \vec{y}_{\mathrm{a}}^{\top} \Delta \vec{u}_{\mathrm{a}}-\Delta \vec{y}_{\mathrm{a}}^{\top} \Delta \vec{u}_{\alpha},
$$

with $\Delta \vec{u}_{\mathrm{a}}=\vec{u}_{\mathrm{a}}^{1}(t)-\vec{u}_{\mathrm{a}}^{2}(t), \Delta \vec{u}_{\alpha}=\vec{u}_{\alpha}^{1}(t)-\vec{u}_{\alpha}^{2}(t)$, and $\Delta \vec{y}_{\mathrm{a}}=$ $\vec{y}_{\mathrm{a}}^{1}(t)-\vec{y}_{\mathrm{a}}^{2}(t)$.

\section{A. Sufficient Conditions for Synchronization in the Non-adaptive Case}

If the adaptation in (8) is not considered, and the breakpoints $L_{k}(t)$ in (7) are constant and equal, i.e. $L_{1}(t)=L_{2}(t)=\ldots=$ $L$, the term $\Delta \vec{y}_{\mathrm{a}}^{\top} \Delta \vec{u}_{\alpha}$ in (13) is positive semidefinite, because $\psi_{\alpha}(\cdot, L): \mathbb{R} \rightarrow \mathbb{R}$ is a nondecreasing function (see Figure 4 ), and therefore

$$
\left[y_{k}^{\mathrm{a} 1}-y_{k}^{\mathrm{a} 2}\right]\left[\psi_{\alpha}\left(y_{k}^{\mathrm{a} 1}, L\right)-\psi_{\alpha}\left(y_{k}^{\mathrm{a} 2}, L\right)\right] \geq 0,
$$

$\forall y_{k}^{\mathrm{a} 1}, y_{k}^{\mathrm{a} 2} \in \mathbb{R}, k \in\{1,2, \ldots, N\}$. In this case, inequality (13) can be rewritten as

$$
\dot{S}_{\mathrm{a}}\left(\Delta \vec{x}_{\mathrm{a}}\right) \leq\left(\alpha-\alpha_{\text {passive }}^{*}\right)\left\|\Delta \vec{y}_{\mathrm{a}}\right\|^{2}+\Delta \vec{y}_{\mathrm{a}}^{\top} \Delta \vec{u}_{\mathrm{a}} .
$$

Noticing that the voltages from the power supplies are the inputs to the electrical network, and the resulting currents are the inputs to the power supply units, i.e. $\vec{u}_{\mathrm{n}}=\vec{y}_{\mathrm{a}}$ and $\vec{u}_{\mathrm{a}}=$ $-\vec{y}_{\mathrm{n}}$, by adding (10) and (14), one has that

$\dot{S}_{\mathrm{n}}\left(\Delta \vec{x}_{\mathrm{n}}\right)+\dot{S}_{\mathrm{a}}\left(\Delta \vec{x}_{\mathrm{a}}\right) \leq-\eta_{\mathrm{s}}\left\|\Delta \vec{y}_{\mathrm{a}}\right\|^{2}+\left(\alpha-\alpha_{\text {passive }}^{*}\right)\left\|\Delta \vec{y}_{\mathrm{a}}\right\|^{2}$.

From this last expression, one can see that if $-\eta_{\mathrm{s}}+$ $\left(\alpha-\alpha_{\text {passive }}^{*}\right)<0$, the time derivative of the positive definite function $V=S_{\mathrm{n}}\left(\Delta \vec{x}_{\mathrm{n}}\right)+S_{\mathrm{a}}\left(\Delta \vec{x}_{\mathrm{a}}\right)$ will be negative semidefinite. Moreover, when $\left\|\Delta \vec{y}_{\mathrm{a}}\right\|>0 \Rightarrow \dot{V}<0$. This will naturally lead to the conclusion that $\left\|\Delta \vec{y}_{\mathrm{a}}\right\| \rightarrow 0$. This result can be stated as follows:

Theorem 3.1: Consider the electrical network with minimal realization (3), satisfying the assumptions in Section II, and an arbitrary number $N$ of power supply units following dynamical reference models defined by (4) to (7), assuming that $L_{1}(t)=$ $L_{2}(t)=\ldots=L_{N}(t)=L, L>0$, with $\alpha>\alpha_{\text {passive }}^{*}$ properly chosen. If $\eta_{\mathrm{s}}$ in (10) satisfies

$$
\eta_{\mathrm{s}}>\left(\alpha-\alpha_{\text {passive }}^{*}\right)
$$

then the power supplies will synchronize without explicit communication among the units.

While a proof of this theorem is available in [15], the corresponding result for the adaptive case remains an open problem.

\section{NUMERICAL EXAMPLES}

\section{A. Without Amplitude Control}

Consider the following parameters for the dynamical reference models in (4), (7):

$$
\begin{aligned}
A_{\mathrm{a}} & =\left[\begin{array}{cc}
0 & \left(\frac{1}{l_{\mathrm{a}}}\right) \\
-\left(\frac{1}{c_{\mathrm{a}}}\right) & -\left(\frac{1}{r_{\mathrm{a}} c_{\mathrm{a}}}\right)
\end{array}\right], \\
B_{\mathrm{a}} & =\left[\begin{array}{c}
0 \\
\left(\frac{1}{c_{\mathrm{a}}}\right)
\end{array}\right], \\
C_{\mathrm{a}} & =\left[\begin{array}{ll}
0 & 1
\end{array}\right], \\
L_{k}(t) & =L,
\end{aligned}
$$


where $l_{\mathrm{a}}=0.001, c_{\mathrm{a}}=1 /\left[l_{\mathrm{a}}(2 \pi 60)^{2}\right], r_{\mathrm{a}}=10$; and $L=10$. This corresponds to an electronic band-pass filter with center frequency $60 \mathrm{~Hz}$, implemented as the parallel combination of an inductor $l_{\mathrm{a}}$, a capacitor $c_{\mathrm{a}}$, and a resistor $r_{\mathrm{a}}$. For this hypothetical electronic circuit, a feasible choice for the storage function is

$$
\begin{aligned}
& S_{k}^{\mathrm{a}}=\frac{1}{2} l_{\mathrm{a}} i_{\mathrm{a}}^{2}+\frac{1}{2} c_{\mathrm{a}} v_{\mathrm{a}}^{2}=\frac{1}{2} l_{\mathrm{a}}\left(x_{1}\right)^{2}+\frac{1}{2} c_{\mathrm{a}}\left(x_{2}\right)^{2}, \\
& \dot{S}_{k}^{\mathrm{a}}=-\frac{1}{r_{\mathrm{a}}} v_{\mathrm{a}}^{2}+\tilde{u}_{k} v_{\mathrm{a}} \leq-\alpha_{\text {passive }}^{*} y_{k}^{2}+\tilde{u}_{k} y_{k},
\end{aligned}
$$

where $x_{1}=i_{\mathrm{a}}$ is the current through the inductor $l_{\mathrm{a}}, x_{2}=v_{\mathrm{a}}$ is the voltage across the capacitor $c_{\mathrm{a}}$. Notice that the agent output is $y_{k}=v_{\mathrm{a}}$, and the input $\tilde{u}_{k}$ to the linear part of each agent is the current into this hypothetical electronic filter. From the above expression for the storage function, we have that $\alpha_{\text {passive }}^{*} \leq \frac{1}{r_{\mathrm{a}}^{2}}=0.01$. The positive feedback introduced as the linear part in (6) would correspond physically to the introduction of a negative resistance $-1 / \alpha$ in parallel to the resistance $r_{\mathrm{a}}$. We have chosen $\alpha=1$ in this case.

Consider an ensemble of $N=3$ agents interconnected as shown in Figure 5, with the following parameters $r_{\mathrm{b}}=0.25 \Omega$, $R_{1}=3 \Omega, L_{1}=1 \mathrm{mH}, C_{1}=100 \mu \mathrm{F}$, and $R_{2}=100 \Omega$.

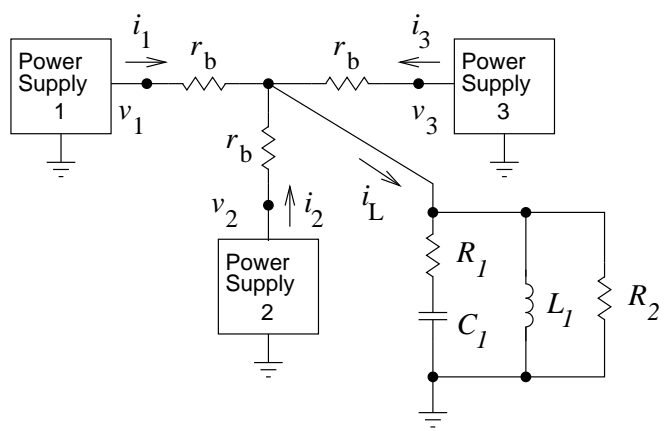

Figure 5. $N=3$ power supply units interconnected by means of a symmetric star-shaped electrical network in order to supply energy to the reactive load represented by $R_{1}, R_{2}, C_{1}$ and $L_{1}$.

In Figure 6 it is shown that the nonlinear oscillatory agents interconnected through the dynamical electrical network are able to attain synchronization of their outputs.

In Figure 7, at $t=0.6 \mathrm{~s}$ the power supply number $k=2$ is removed from the system. This result shows that the remaining power supplies continue to be synchronized, but the voltage regulation in the bus is very poor.

\section{B. With Amplitude Control}

In this section, the same set of parameters used for the example in Section IV-A will be employed, but instead of a fixed value $L$ for the breakpoints $L_{k}(t)$ in (7), the following amplitude control parameters were chosen for the dynamics in (8): $K_{\mathrm{p}}=2, K_{\mathrm{i}}=10, v_{\text {ref }}=\frac{100}{\sqrt{2}}, \tau=0.1$.

The effect of the adaptation law (8) on the synchronization of the power supplies can be seen in Figure 8. The initial conditions of the dynamics reference models were kept the same as in the previous simulation depicted in Figure 6, which are far from steady-state values, and this partially explains the great amplitude variations observed in this initial phase (from $t=0$ to $t \approx 0.4 \mathrm{~s}$ ), despite the fact that the power supplies synchronize much faster than this initial transient period. (a)

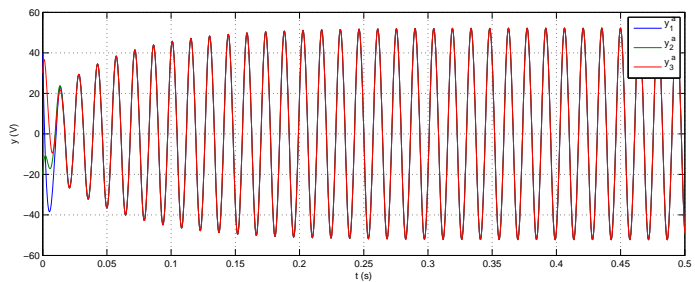

(b)

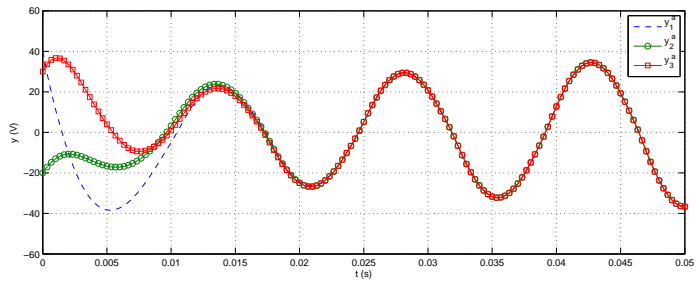

Figure 6. Simulated results for the case shown in Figure 5. (a) $N=3$ power supplies departing from different initial conditions have their outputs synchronized. (b) Detail.

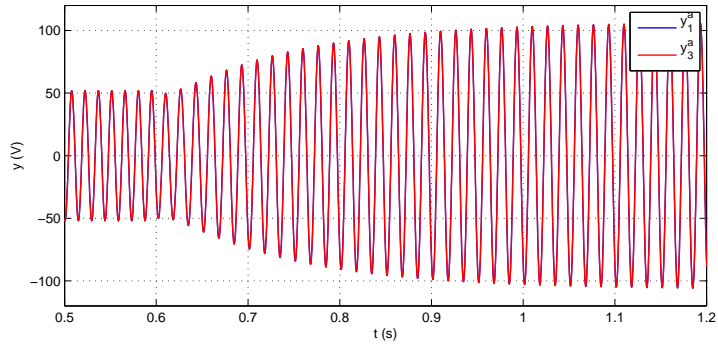

Figure 7. Simulated results for the case shown in Figure 5, corresponding to the continuation of the results shown in Figure 6, with abrupt removal of the power supply number 2 at $t=0.6 \mathrm{~s}$. Power supplies 1 and 3 remain synchronized, but the bus voltage is greatly afftected because there is no amplitude control.

In Figure 9 the simulated results for the case of abrupt removal of power supply number 2 at $t=0.6 \mathrm{~s}$, with amplitude control activated, are presented. It can be seen that the amplitude control was effective in returning the RMS bus voltage to the reference value $v_{\text {ref }}$. It is important to notice, however, that the adjustment of the amplitude controllers parameters $K_{\mathrm{p}}$ and $K_{\mathrm{i}}$ could lead to better results, such as a smaller settling time associated with the amplitude variations after $t=0.6 \mathrm{~s}$.

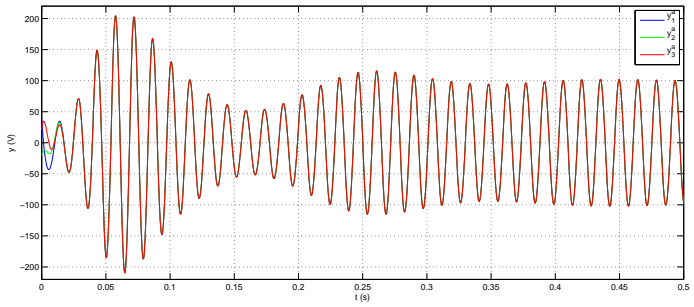

Figure 8. Simulated results for the case shown in Figure 5, with amplitude control (8) activated such that, after an initial transient, the amplitude of each power supply output voltage is kept at $100 \mathrm{~V}$. 


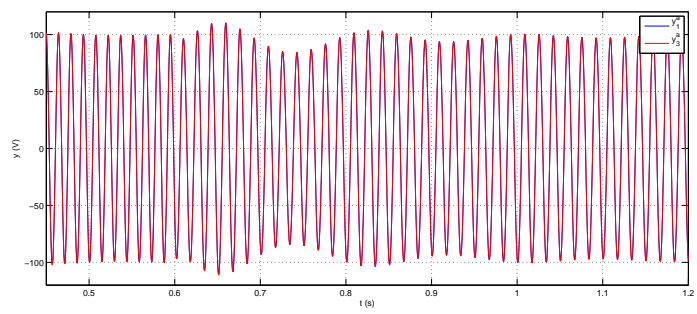

Figure 9. Simulated results for the case shown in Figure 5, with abrupt removal of the power supply number 2 at $t=0.6 \mathrm{~s}$. Power supplies 1 and 3 remain synchronized, and the bus voltage is kept at $100 \mathrm{~V}$ using the amplitude control (8).

\section{Conclusions}

We considered the synchronization of power supplies in an isolated grid for which the primary energy sources are interfaced with the power bus using power electronic inverters. We explored the flexibility provided by these devices and novel results regarding the synchronization of dynamical systems to construct voltage power sources that naturally synchronize with each other without explicit communication. Our main result proves that the power sources asymptotically synchronize in the absence of voltage amplitude control. A similar analysis that is valid under amplitude control remains an open problem and it is currently under investigation.

\section{ACKNOWLEDGMENTS}

The first author express thanks to the Universidade Federal de Minas Gerais, and to the Conselho Nacional de Desenvolvimento Científico e Tecnológico - CNPq, Brazil, for financial support under grant 200312/2010-3.

\section{REFERENCES}

[1] L. Alberto, F. Silva, and N. G. Bretas, "Direct methods for transient stability analysis in power systems: state of art and future perspectives," in IEEE Power Tech Proceedings, vol. 2, 2001.

[2] F. Katiraei and M. R. Iravani, "Power management strategies for a microgrid with multiple distributed generation units," IEEE Trans. on Power Systems, vol. 21, no. 4, pp. 1821-1831, 2006.

[3] E. Coelho, P. Cortizo, and P. Garcia, "Small-signal stability for parallel-connected inverters in stand-alone AC supply systems," IEEE Trans. on Industry Applications, vol. 38, no. 2, pp. 533-542, 2002.

[4] F. Dörfler and F. Bullo, "Synchronization and transient stability in power networks and non-uniform Kuramoto oscillators," in ACC 2010 Proceedings. IEEE American Control Conference, 2010, pp. 930-937.

[5] A. Mohd, E. Ortjohann, D. Morton, and O. Omari, "Review of control techniques for inverters parallel operation," Electrical Power Systems Research, vol. 80, pp. 1477-1487, 2010.

[6] A. Abbondanti, "Arrangement of parallel static AC power sources proportions," United States Patent - 3,864,620, February 1975.
[7] T. Kawabata and S. Higashino, "Parallel operation of voltage source inverters," IEEE Trans. on Industry Applications, vol. 24, no. 2, pp. 281-287, 1988.

[8] A. Tuladhar, H. Jin, T. Unger, and K. Mauch, "Parallel operation of single phase inverter modules with no control interconnections," in Applied Power Electronics Conference and Exposition - APEC '97 Conference Proceedings, vol. 1, feb. 1997, pp. 94-100.

[9] E. C. Furtado, L. A. Aguirre, and L. A. B. Tôrres, "UPS parallel balanced operation without explicit estimation of reactive power: A simpler scheme," IEEE Trans. on Circuits and Systems II - Express Briefs, vol. 55, no. 10, pp. 1061-1065, 2008.

[10] M. Arcak, "Passivity as a design tool for group coordination," IEEE Trans. on Automatic Control, vol. 52, no. 8, pp. 1380-1390, 2007.

[11] G.-B. Stan and R. Sepulchre, "Analysis of interconnected oscillators by dissipativity theory," IEEE Trans. on Automatic Control, vol. 52, no. 2, pp. 256-270, 2007.

[12] A. Pogromsky and H. Nijmeijer, "Cooperative oscillatory behavior of mutually coupled dynamical systems," IEEE Trans. on Circuits and Systems - I: Fundamental Theory and Applications, vol. 48, no. 2, pp. 152-162, 2001.

[13] B. Brogliato, R. Lozano, B. Maschke, and O. Egeland, Dissipative Systems Analysis and Control - Theory and Applications, 2nd ed. Springer, 2007.

[14] B. D. O. Anderson and S. Vongpanitlerd, Network Analysis and Synthesis: A Modern Systems Theory Approach. Prentice-Hall, Inc., 1973.

[15] L. A. B. Torres, J. P. Hespanha, and J. Moehlis, "Passivity based synchronization of voltage power supplies - a constructive approach," 2011, to be submitted. 